\title{
Adsorption behavior of multicomponent protein mixtures containing a1-proteinase inhibitor with the anion exchanger, 2-(diethylamino)ethyl-Spherodex
}

\begin{abstract}
The equilibrium binding behavior of Ŭl-proteinase inhibitor (Ŭl-PI) in the presence of human serum albumin (HSA) has been determined in packed bed systems with the anion exchanger, 2-(diethylamino)ethyl (DEAE)-Spherodex. Experimental data derived for the individual proteins were compared with the corresponding data obtained from batch adsorption studies as well as studies in which mixtures of these two proteins were loaded at different concentration ratios onto columns of the same anion exchange adsorbent. The results confirm that Ŭl-PI has a greater affinity for the anion exchanger, although competitive adsorption was observed as the inlet concentration of HSA was increased. Under these conditions, decreased binding capacities and lower dynamic adsorption rates were observed for ÜPI with the DEAE-Spherodex anion exchange adsorbent. The results are discussed in terms of the influence which various contaminants that occur in multicomponent mixtures of proteins from human plasma can have on the equilibrium binding characteristics of a target protein with weak or strong ion exchange adsorbents under conditions approaching concentration overload in preparative chromatographic systems. These investigations have also addressed, as the first part of an iterative approach for the simulation of the adsorption behavior of multicomponent mixtures of human plasma proteins with ion exchange and affinity chromatographic adsorbents, the ability of noncompetitive and competitive Langmuirean models to simulate the adsorption of Ŭl-PI in the presence of different concentrations of HSA to DEAE-Spherodex.
\end{abstract}

Keyword: Adsorption; Ŭl-proteinase inhibitor (Ŭl-PI); Anion exchanger 